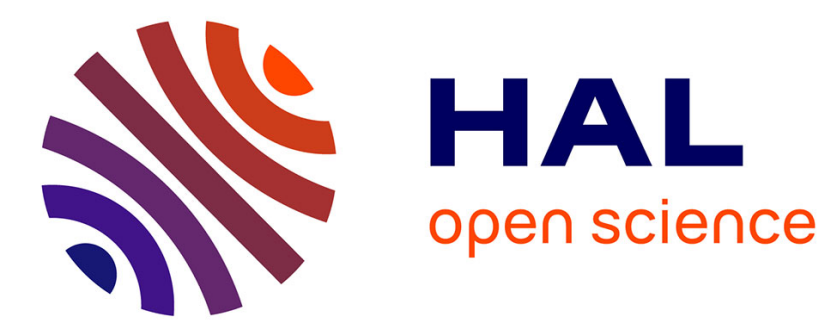

\title{
Closing the loop between body compensations and upper-limb prosthetic movements: a feasibility study
}

Mathilde Legrand, Nathanael Jarrasse, Etienne de Montalivet, Florian Richer, Guillaume Morel

\section{- To cite this version:}

Mathilde Legrand, Nathanael Jarrasse, Etienne de Montalivet, Florian Richer, Guillaume Morel. Closing the loop between body compensations and upper-limb prosthetic movements: a feasibility study. IEEE Transactions on Medical Robotics and Bionics, 2021, pp.1-1. 10.1109/TMRB.2020.3048251 . hal-03099461

\section{HAL Id: hal-03099461 \\ https://hal.science/hal-03099461}

Submitted on 15 Feb 2021

HAL is a multi-disciplinary open access archive for the deposit and dissemination of scientific research documents, whether they are published or not. The documents may come from teaching and research institutions in France or abroad, or from public or private research centers.
L'archive ouverte pluridisciplinaire HAL, est destinée au dépôt et à la diffusion de documents scientifiques de niveau recherche, publiés ou non, émanant des établissements d'enseignement et de recherche français ou étrangers, des laboratoires publics ou privés. 


\title{
Closing the loop between body compensations and upper-limb prosthetic movements: a feasibility study
}

\author{
Mathilde Legrand ${ }^{1}$, Nathanaël Jarrassé ${ }^{1}$, Etienne de Montalivet $^{1}$, Florian Richer ${ }^{1}$ and Guillaume Morel ${ }^{1}$
}

\begin{abstract}
To control the robotic joints of an upper limb prosthesis, most existing approaches rely on decoding the user motor intention from electrophysiological signals produced by the subject, and then executing the desired movement. This suffers from important limitations and requires extended training, particularly when a large number of prosthetic joints have to be controlled. Even when they master the control of their prosthesis, many amputees underuse the prosthetic mobility to the benefit of compensatory body movements, whose generation is less expensive and more natural from a cognitive point of view. Indeed, with an arm prosthesis, hand movements result from a combination of human and robotic joint motions.

We propose in this paper to use these compensatory motions as an error signal to servo the robotic controller. This approach thus creates a coupling between body compensations and prosthetic movements.

To study the feasibility of such a coupling, the concept is tested with ten able-bodied subjects wearing an emulated elbow prosthesis and one congenital arm amputee. The results validate the concept, which allows naive subjects to control the prosthetic joint with no or very short training period.
\end{abstract}

Index Terms-Physical human-robot interaction, prosthetics and exoskeletons, human-in-the-loop and body compensations.

\section{INTRODUCTION}

$\mathbf{R}$ OBOTIC prostheses aim to help amputated people recovering motor capabilities and thus functional independence. In recent years, the development of advanced mechatronic prostheses has led to technological improvements such as a better fixation system to the body with osseointegration [1] or a larger number of degrees of freedom (DOF) [2]-[4]. However, providing these devices with a natural and efficient control is still a major challenge [5]-[7].

Most control approaches use a direct connection between an auxiliary signal produced by the subjects (like electromyographic signal) and the resulting movement of the prosthesis generated by the controller (see Auxiliary Signal Control Figure 1(a)). Examples of this approach include conventional myoelectric control, integrated into most of the commercially available devices. With electromyogram sensors, signal processing suffers from a lack of robustness to numerous factors [6], including electrodes placement, environmental conditions, muscular fatigue or human variability in myoelectric activity.

This work was supported by the ANR-BYCEPS, ANR-18-CE19-0004.

1 The authors are with the Institute for Intelligent Systems and Robotics, Sorbonne Université, Paris, with CNRS, UMR7222 and with INSERM U1150. e-mail: \{mathilde.legrand, nathanael.jarrasse, guillaume.morel\}@ sorbonneuniversite.fr

This paper has supplementary downloadable multimedia material provided by the authors. This includes a MP4 video that shows the experimental set-up and part of the results. This material is $7.2 \mathrm{MB}$ in size.
Therefore, to achieve enough robustness, the subject is asked to send high intensity sequential signals and/or co-contraction sequences, that ease the motor intention recognition. As a result, controlling the prosthesis requires a long learning phase [5], [8], [9], for a limited control dimensionality. Literature reports attempt to use other signals and treatments, such as cerebral state [10], [11], or head or foot movements [12][15]. However, none of these solutions seem to significantly increase the easiness of control [7]. These issues are becoming particularly problematic with the increasing number of prosthetic DOF to control with recent prostheses.

Artificial Intelligence is a possible solution to ease control. The idea is to let the robot learn how to decode patientspecific control signals, rather than let the subject learn how to control the prosthesis. For example, in [16] and [17], pattern recognition-based myoelectric control is used for decoding natural muscular synergies. However, again, robustness issues occur and limit the number of patterns that the algorithm can really recognize during daily life scenarios.

Recently, alternative solutions have been explored to decrease the number of patterns to be identified, without impacting the movement ability of the prosthesis. They propose to separate the control of hand (grasping function) and wrist/elbow joints (reaching, positionning functions), considering that myoelectric approaches should be used to manage the grasping functions of the hand, but not being overloaded with the control of the other intermediate joints. To move the prosthetic wrist and/or elbow, these approaches avoid asking subjects to generate an extra signal indepently from the control of their healthy joints. Instead, they consist in observing the motion of the healthy body and then in deducing the completing motion of the prosthesis. The input signal of the controller is not an auxiliary signal but the kinematic measurements of the body motion (see Motion Completion Control Figure 1(b)). Examples of this approach include joint synergy-based control schemes that map the kinematic body measurements into prosthesis movements, through models of natural joint coordinations [18], [19]. Yet, synergies, be it for muscles or joints, are task-dependent [20], [21]: each task requires its own model. The control law must then contain a model for each type of motion, which leads to complex algorithm structures. Synergy-based controls are thus limited to a small set of motions.

We propose in this paper a new control approach exploiting measurements of body position in a different way. It tackles a common limitation to all existing prosthetic control schemes: the fact that errors have to be corrected by the user, mainly through visual feedback, requiring a heavy mental workload 


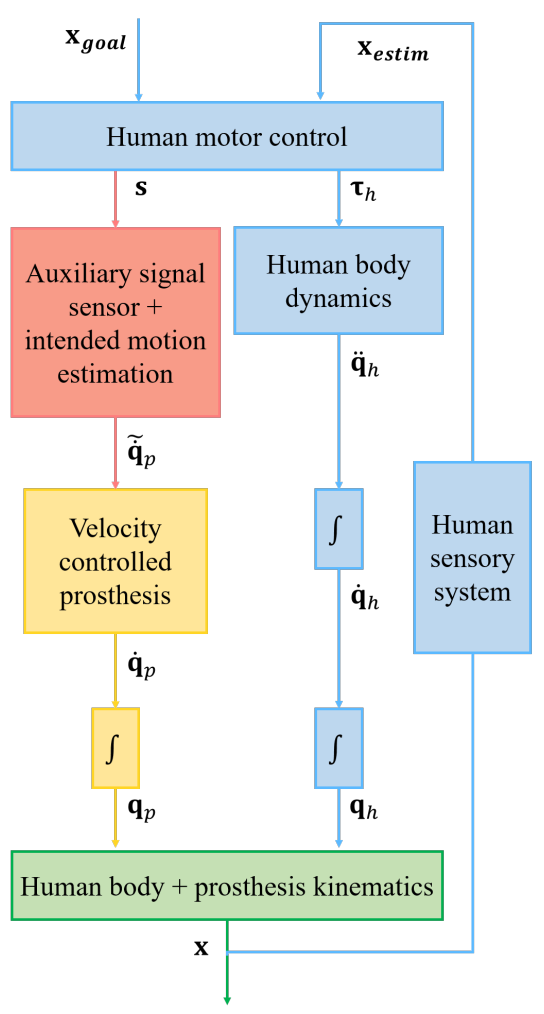

(a) Auxiliary Signal Control

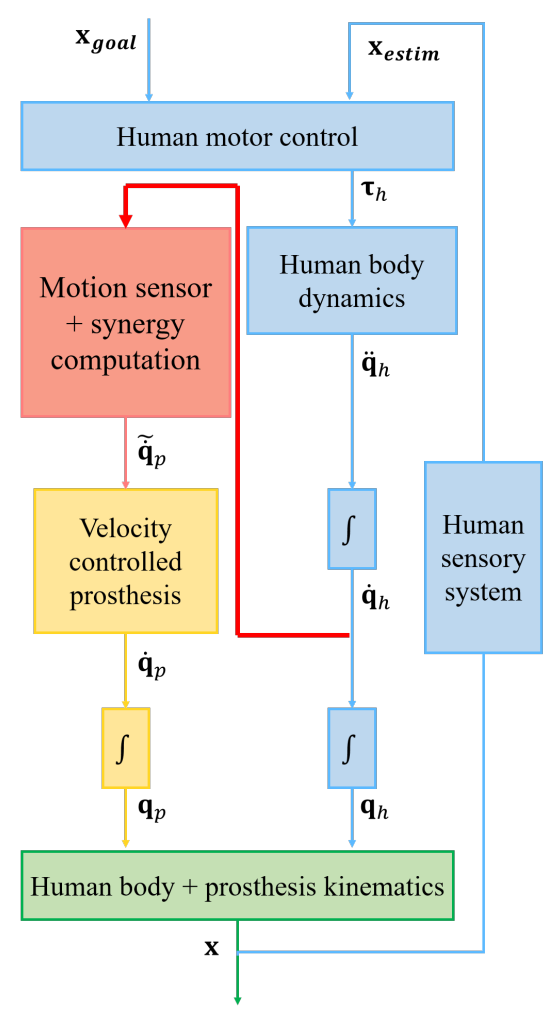

(b) Motion Completion Control

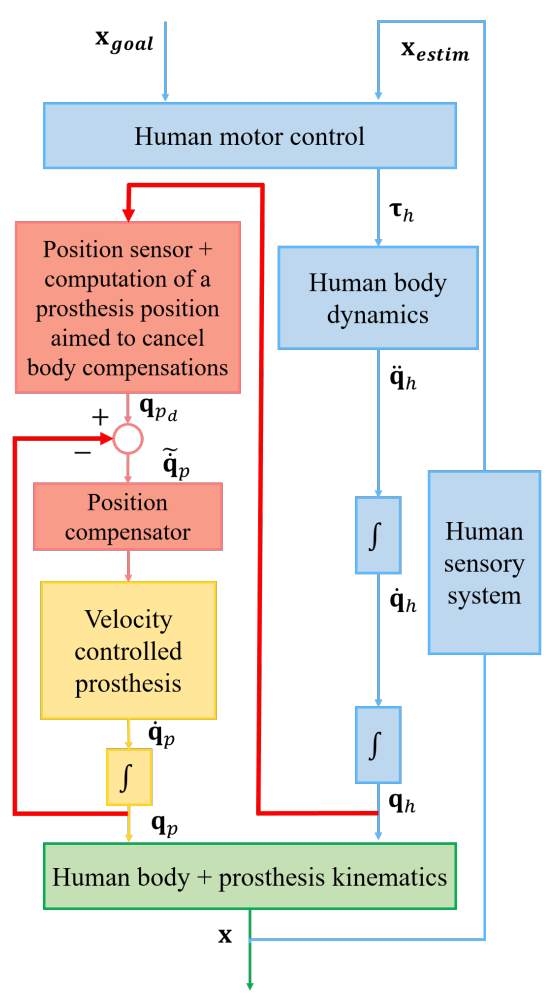

(c) Compensations Cancellation Control

Fig. 1: Control diagram of prosthesis control schemes. Bold and red arrows indicate the main differences from the Auxiliary Signal Control. (a) Mainstream approach: the human subject is asked to control the robotic device by generating auxiliary signals, that are supposed to be independent of the control of the human parts of the arm. (b) The controller input is the body motion of the user which is completed by the prosthesis motion with joints synergy models. (c) The user is in charge

of the end-effector task while the prosthesis control focuses on the human posture, in order to detect whether body compensations are employed and to cancel them when necessary.

and increasing the time of the task [7]. It operates in three steps: (i) analysis of the body posture to evaluate whether the subject is currently compensating for an inadequate prosthesis configuration; (ii) when a body compensation is detected, computation of a new desired position of the prosthesis that cancels the compensation; (iii) servoing the prosthesis joints position to this desired value with a secondary loop. Figure 1(c) sketches the corresponding diagram of this control scheme, later called Compensations Cancellation Control (CCC). Note that, like Motion Completion Control, this work focus on intermediate joints control and not on hand grasping functions, since those are hardly redundant and cannot be compensated by other body joints.

In this paper, we propose to study the feasibility of this concept by implementing it for the control of one prosthetic joint. In Section II, the general approach is further described before being detailed for the control of a prosthetic elbow. In Section III, we present the experimental set-up developed for the different validation experiments. In Section IV, we discuss the tuning of the controller from an experimental analysis. In Section V, we evaluate the response of human-robot pairs: ten able-bodied subjects perform a path tracking task, while wearing an emulated prosthetic elbow that drove their motions.
The approach is then evaluated with a congenital arm amputee in Section VI.

\section{Compensations CANCEllation Control}

\section{A. General concept}

The concept we are proposing is based on the observation of a natural behaviour in subjects wearing a prosthesis: the body compensations. These movements are typically produced by amputees who wear a passive (non-mobile) prosthesis, to position their hand in spite of the lack of distal mobility. For instance, if a prosthetic elbow joint is not extended enough while the user wants to reach a distant target, the user will lean forward using the trunk to achieve the goal. Body compensations are well known in the medical community and are to be avoided as they may generate fatigue or even musculoskeletal disorders [22], [23].

Interestingly, body compensatory motions are also frequently observed in amputees who wear a robotic myoelectric prosthesis [24]-[26]. We hypothesize that this results from the high cognitive cost when using the prosthetic joints. Indeed, as observed in Figure 1(a), Auxiliary Signal Control creates two parallel loops for the subject, which are in conflict: the natural motor control loop (control of healthy joints, in blue) and the 
prosthesis motion loop (red and yellow). Since generating an individual myoelectric signal is not natural for the Central Nervous System, the subject naturally tends to use the motor control loop to perform a task: s/he tends to use only residual functional joints (through body compensations), abandon the active dimension of the prosthesis and use it as a rigid tool. We propose to take inspiration from this natural reaction to build a relevant signal expressing the prosthesis positioning error, error to which the device is then continuously servoed. For example, when a subject is leaning, it can be inferred that the elbow is not extended enough and the robot controller will extend it. Since the natural focus of the subject is the hand position, we assume that the subject will react to the elbow extension and, to reach the intended target, will lean less. The continuous prosthesis servoing on body compensations creates a human-robot coupling which allows to reduce user body compensations with prosthesis motions, while achieving a given task (see Figure 1(c)).

It can be noticed that, like Motion Completion Control, CCC does not require the subject to generate auxiliary signals. Moreover, as postural compensations are naturally employed by the Central Nervous System, we hypothesize that the user would master the control of the prosthesis without any previous knowledge on the concept.

Note that controlling a robot from the body configuration of its user is not a completely new concept. It has been explored in the literature in the context of cobotics, for industrial applications [27]-[29]. Significant differences exist with our approach since we propose to control a generic prosthesis: because it aims at replacing a human limb, it must move in accordance with the rest of the human body. This idea was first presented in [30] in which it was verified that using postural compensations as control input does not heighten them. The work of the aforementioned paper is extended here with a detailed explanation of CCC concept, a presentation of an experimental tuning approach, an extensive study of human's response to the prosthesis servoing and a further validation experiment with a congenital amputee participant.

Implementing the concept of $\mathrm{CCC}$ raises three main questions:

1) in everyday life, how to continuously measure the posture of the user and identify a postural compensatory movement out of this measure?

2) how natural is the prosthesis manipulation for a naive subject, or more precisely, is a naive subject able to use a prosthesis equipped with $\mathrm{CCC}$ without any explanations on the concept?

3 ) is the tuning of the controller valid for every subject or will a personalization be required?

In this paper, we focus on the second and third questions which are addressed in Sections V and VI, while Section IV presents the tuning of the controller with one subject. The first question is bypassed thanks to the design of a specific experimental setup where the detection and identification of compensatory movements can be easily implemented.

In the very next Section II-B, CCC implementation for the case study considered in this work - controlling a prosthetic elbow - is detailed.

\section{B. Control formulation for an example application}
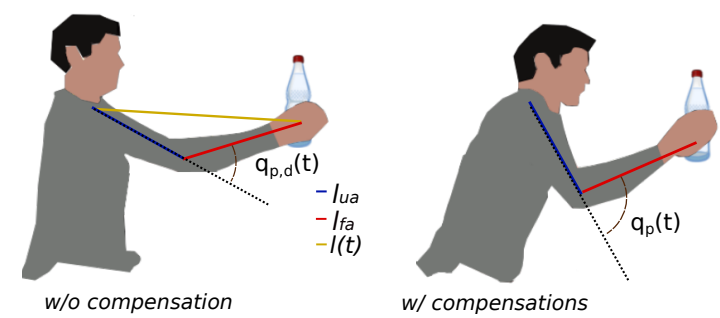

Fig. 2: Anatomical parameters definition for the control law

In the rest of the paper, in order to validate the concept described above, CCC is implemented to control a prosthetic elbow joint (flexion/extension). To formulate the control law, we need (i) to compute a desired position correction for the prosthesis, corresponding to an identified body compensation and (ii) to design a compensator that servoes the prosthetic device to this position. In the particular case considered here, the first step is easily solved: the acromion is considered to encode the postural compensation of the subject since its motion reflects the motions of both the trunk and the scapula, the main compensatory joints in upper-limb movements [25], [26], [31]. From the measurement of the acromion position in a reference (non-compensatory) state and the end-effector position in current state, we can directly compute a desired position for the prosthesis, $q_{p, d} \in \mathbb{R}$, corresponding to a cancellation of the acromion displacement.

We first estimate $l(t)$, the distance between the acromion position in the reference posture and the end-effector position: $l(t)=\left\|\mathbf{x}_{E E}(t)-\mathbf{x}_{A_{\text {ref }}}\right\|$ where $\|$.$\| is the euclidian norm,$ $\mathbf{x}_{E E}(t)$ is the current position of the end-effector and $\mathbf{x}_{A_{\text {ref }}}$ is the reference position of the acromion, in the global laboratory frame. Given the distance $l(t)$, and knowing $l_{u a}$ and $l_{f a}$ the lengths of the subject's upper-arm and forearm (see Figure 2), standard inverse kinematics yields:

$$
q_{p, d}(t)=\pi-a \cos \left(\frac{l_{f a}^{2}+l_{u a}^{2}-l(t)^{2}}{2 l_{u a} l_{f a}}\right)
$$

Note that this computation requires to measure the user limb lengths and to integrate them into the controller parameters. To servo the position of the prosthesis to this value, a controller enforces a first order closed-loop dynamics for its angle, $q_{p}(t)$ :

$$
\tilde{\dot{q}}_{p}(t)=\lambda Z_{q_{0}}\left(q_{p, d}(t)-q_{p}(t)\right)
$$

where $q_{p, d}(t)$ is the desired prosthetic elbow angle (computed to enable a non-compensatory posture for the user) and $\lambda$ is a scalar gain that tunes the rate of correction. $Z_{q_{0}}()$ is a dead-zone function defined by:

$$
\forall \epsilon \in \mathbb{R}, Z_{q_{0}}(\epsilon)=\left\{\begin{array}{l}
0 \text { if }|\epsilon| \leqslant q_{0} \\
\epsilon-\operatorname{sign}(\epsilon) q_{0} \text { otherwise }
\end{array}\right.
$$

where $q_{0}$ is a threshold, that can be added for more comfort of the user.

Contrary to usual prosthetic control, where the user is in charge of the prosthetic joints position, this implementation considers that the user is in charge of the end-effector position via body 
motions, while the prosthesis is responsible for the correct user's posture.

It can be noticed that this controller involves the tuning of only two scalar parameters: $\lambda$, that tunes the bandwidth of the secondary loop, and $q_{0}$ that tunes the acceptable error. The tuning of these parameters was performed experimentally, as exposed Section IV.

\section{Materials And Methods}

The feasibility study presented in this paper gathers three complementary experiments. Each of them were carried out in accordance with the recommendations of Université Paris Descartes ethic committee CERES, which approved the protocols (NIRB: 20163000001072). The participants gave their informed consent, in accordance with the Declaration of Helsinki.

\section{A. Hardware}

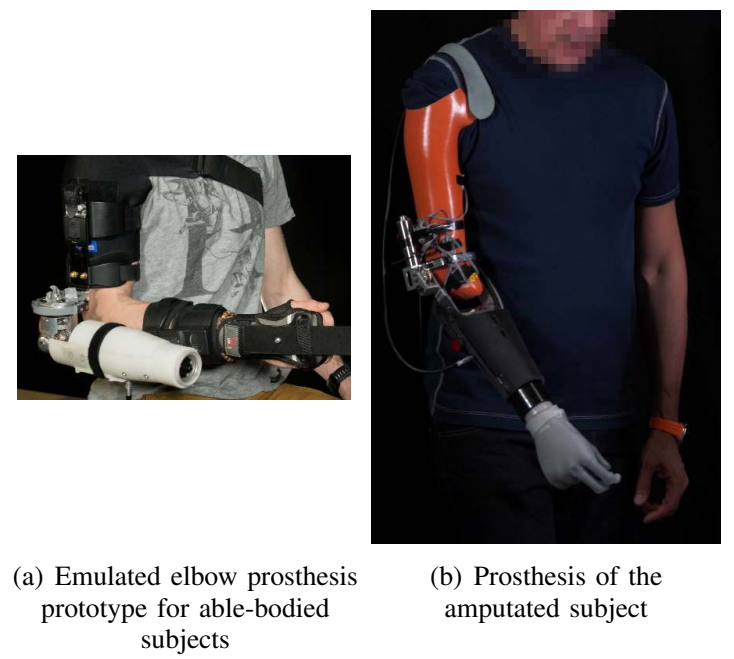

Fig. 3: Prosthesis prototypes (a) mounted on an able-bodied subject's arm, in such a way that his elbow motions are totally governed by the prosthesis; (b) for the amputated subject, for which only the elbow joint was actuated.

The first two experiments aim at tuning CCC and evaluating its potential. They were performed with able-bodied participants, aged 20-24, with no previous experience on prosthetic devices. A prosthesis prototype was adapted to imitate the wearing of a prosthesis. The prototype was a 1-DOF robotic joint (actuated by a 9W Faulhaber 2232U006S, fitted with 1:1000 gear ratio reducer) controlled by a Raspberry PI, through a DC motor driver running a low level velocity control loop. The angular velocity was limited to $50 \mathrm{deg} . \mathrm{s}^{-1}$ because of the actuation capacities. The device was fixed on an orthosis, attached to the participants' arm (see Figure 3(a)). The elbow was not backdriveable so that elbow motions of the subjects were only due to prosthesis movements. The third experiment, that aims to validate the use of CCC by final-end users, was performed with a congenital amputee, wearing his own prosthesis which has a motorized elbow (see Figure 3(b)).

\section{B. Experimental methodology}

During the experiments, subjects were sitting and were asked to follow a moving target in their sagittal plane. In order to observe whether they were compensating or not, acromion and end-effector positions were recorded with the motion capture system OptiTrack (NaturalPoint Inc.). These positions were transmitted in real time to the prosthesis during the experiment, to implement the proposed controller.

At the beginning of the experiment, the reference acromion position, defined as the initial position, was recorded while the subjects were sitting with a vertical torso and the upper arm along their body. The definition of a more global reference body configuration falls under the question of the identification of compensatory motions, which is not the main purpose of this work and will be discussed in Section VII. To evaluate whether CCC was natural or not to the subjects, no instructions nor any explanation on how it works were given to any subject. They were only told that they had to focus on the achievement of the task (positioning their end-effector) and that the prosthetic device would "move to try helping them". The trajectories to follow for the different experiments were materialized with a WAM ${ }^{\circledR}$ Arm (Barrett Technology) always referred to WAM ${ }^{\mathrm{R}}$ in the following; robot and robotic being sometimes employed for the prosthetic devices-. Specific details of each experimental protocol are given in the corresponding sections.

\section{Tuning of Compensations CANCELlation CONTROL}

Using body compensations as control input for the prosthesis creates a coupling between human and robotic motions: when the subject uses a body compensation to position his/her hand, this generates a robotic motion which, in turn, is expected to induce a correction of the body posture on the human side. It is therefore likely that the tuning of the parameters $\lambda$ and $q_{0}$ influences transient response and stability. In a standard engineering process, a stability analysis should be performed to determine desirable values for these parameters. However, with CCC, the human motor control system is part of the loop. Therefore, it is quite a challenging issue to derive a model that could be used reliably for such a theoretical analysis. In order to get around this difficulty, we experimentally investigated the tuning of $\lambda$ and $q_{0}$ by observing the effect of their variation on the transient response.

\section{A. Protocol}

One able-bodied subject participated in the experiment. She had to follow a target moving along a $20 \mathrm{~cm}$ straight line, carried by the WAM ${ }^{\mathrm{R}}$ Arm. When arrived at one extremity of the line, the WAM ${ }^{\circledR}$ paused for 2 seconds before leaving again in the opposite direction.

First, we studied the influence of the gain $\lambda$ without the possibility to stop the prosthesis within a deadzone: $q_{0}$ was set to $0 \mathrm{deg}$ in such a way that $\lambda$ is always active. The prosthesis motions were controlled with CCC, with $\lambda \in\{0.4 ; 2 ; 10\}\left[s^{-1}\right]$ successively. For each value of $\lambda$, the task was repeated five 


\section{Elbow angular velocity}
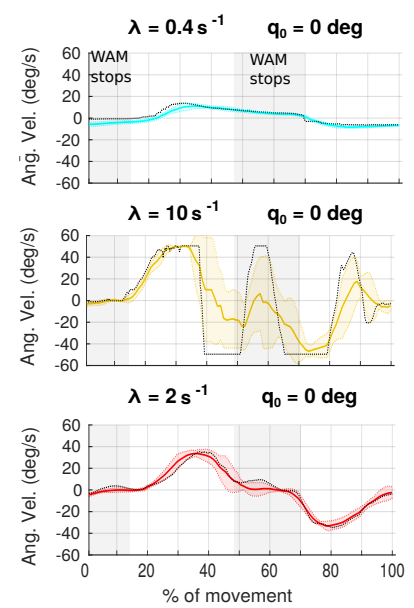

(a) Gain impact on the stability of the system

\section{Elbow angular velocity}
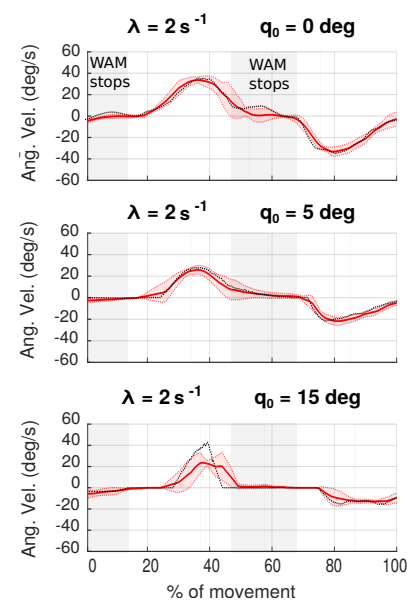

(b) Influence of the threshold

Fig. 4: Mean and standard deviation of elbow angular velocity profile and of acromion displacement (body compensations) during a back and forth of the WAM ${ }^{\circledR}$ Arm. Black dashed lines are examples of one trial for the corresponding $\lambda$. Grey areas are when the WAM ${ }^{\circledR}$ Arm stops before leaving again.

times. The reference posture was defined as the initial position of the subject.

In a second time, $\lambda$ was fixed to its optimal value and $q_{0}$ was set to 5 and 15 deg to evaluate its impact on compensatory motions reduction. Here again, five trials were performed for each value of $q_{0}$.

\section{B. Results and analysis}

Human acromion displacement and elbow velocities are observed in order to evaluate their coupled dynamic evolution. Figure 4(a) shows the mean and standard deviation of the prosthetic elbow angular velocity and the acromion displacement over the five trials, for the different values of $\lambda$ and $q_{0}=0$ deg. When $\lambda=0.4 s^{-1}$ (first row, cyan), the elbow velocity command remains small, leading to a slow movement of the elbow. As the prosthesis is not reactive enough, the subject largely mobilizes compensatory movements to perform the task, which can be observed in the corresponding acromion displacement plot: the maximum displacement measured in the mean plot is close to $15 \mathrm{~cm}$. When $\lambda=10 \mathrm{~s}^{-1}$ (second row, yellow), the magnitude of the compensatory acromion displacement is drastically reduced $(5 \mathrm{~cm}$, at most, for the mean plot). However, the velocity command is jerky and quickly reaches high values, as illustrated with the black line plot corresponding to an example trial. Notice that the mean plot smooths this oscillatory behavior as the timereproducibility between trial is low: the velocity commutes at different times between trials, leading to large variability. All in one, the movement of the prosthesis is not mastered, as it oscillates even when the WAM arm is still (gray areas). With $\lambda=2 s^{-1}$ (last row, red), the velocity profile is much gradual and smoother. The standard deviation is small and the subject can monitor the prosthesis motion. Yet, the subject does not mobilize large compensations, as the maximal acromion displacement, for the mean plot, is limited to about $7.5 \mathrm{~cm}$. Note that this value of $\lambda$ corresponds to a frequency $f=\frac{\lambda}{2 \Pi} \approx$ $0.32 \mathrm{~Hz}$, one order of magnitude smaller than the frequency of the human hand position controller (which ranges from 2 to $5 \mathrm{~Hz}$ according to [32]). As viewed by the human controller, the prosthesis' position can thus be considered as a slowly varying - or quasi-static - disturbance. In the rest of the paper, we thus selected $\lambda=2 s^{-1}$.

Note that with $q_{0}=0 \mathrm{deg}$, the robotic device never stops in practice. Indeed, the subject is never totally still with a perfectly null acromion displacement. This can be seen in Figure 4(a), where the velocity commands hardly stay at zero when the WAM does not move. A perfectly null deadzone necessarily leads to a permanent (even slow) oscillation of the prosthesis, which is not desirable. A non-null deadzone has thus to be added so that the device can stop without requiring the subject to be exactly at the reference position. Yet, a too high threshold will increase the zero-velocity zone around the reference posture, which may lead to higher acromion displacement for a same prosthesis position. Two values of $q_{0}$ were tested: 5 and $15 \mathrm{deg}$. Figure 4(b) shows that, compared to $q_{0}=0 \mathrm{deg}$ (first row), $q_{0}=5 \mathrm{deg}$ (middle row) only increases the acromion displacement by $3 \mathrm{~cm}$ while slightly decreasing the elbow joint velocity, but without altering the motion dynamic. With $q_{0}=15 \mathrm{deg}$ (last row), the elbow angular velocity maximum is similar to the one with $q_{0}=5$ deg but with higher acromion displacement $(+5 \mathrm{~cm}$ and $+8 \mathrm{~cm}$ compared to $q_{0}=5 \mathrm{deg}$ and $q_{0}=0 \mathrm{deg}$ respectively). The motion dynamics is also affected since the temporal evolution of the elbow angular velocity is less smooth.

In the rest of the paper, a threshold of $q_{0}=5 \mathrm{deg}$ is selected, for all the subjects. To further support this choice, it can be noticed that a gap of $5 \mathrm{deg}$ is never considered as uncomfortable in posture assessments (see [33], [34] for instance). 


\section{EVALUATION OF COMPENSATIONS CANCELLATION CONTROL}

Following the tuning of CCC controller, the two questions listed in Section II-A can be further addressed:

1) is a naive subject able to use a prosthesis equipped with CCC without any explanations on the concept?

2 ) is the tuning of the controller valid for every subjects or will a personalization be required?

To provide answers, we conducted a detailed study on ten participants who performed a path tracking task. Ten ablebodied subjects wearing the emulated prosthetic elbow were involved. As stated Section III-B, no explanation about CCC were given to any subject to study intuitiveness (first question); $\lambda$ and $q_{0}$ parameters were the same for all participants to answer the second question.

\section{A. Protocol}

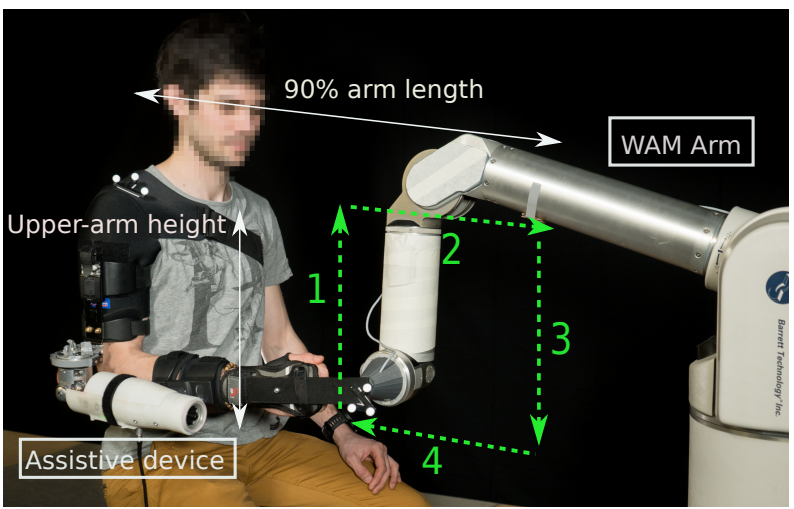

Fig. 5: Path tracking task. Dimensions of the rectangle are adjusted to the subject's morphology. Numbers 1 to 4 correspond to the movement stages. The WAM ${ }^{\circledR}$ Arm pauses briefly at each corner.

Subjects were asked to perform a path tracking task: they had to follow a material target carried by the WAM $^{\circledR}$ Arm, that slowly moved and drew a rectangle in their sagittal plane. The dimensions of the rectangle were adapted to the subjects' morphology and the initial position was the upper-arm along the trunk and the elbow flexed at 90 deg (see Figure 5). The path tracking task was deliberately made slow to ensure a progressive reconfiguration of the subjects' posture during the task.

The prosthetic elbow was successively controlled by three modes:

1) natural (mode $\mathrm{N}$ ), without wearing the device. This was used as a reference;

2) with the elbow joint Locked at $90 \mathrm{deg}$ (mode L), preventing from any movement of the elbow. This mode was chosen to evaluate the magnitude of compensations when the elbow is inoperative;

3) Compensations Cancellation Control (CCC): $q_{0}$ was set to $5 \mathrm{deg}$ and $\lambda$ to $2 \mathrm{~s}^{-1}$

A full session included five consecutive trials per mode $((5$ trials $) \times 3$ modes $=15$ trials in total $)$. For CCC, there were also ten training trials, whose data are not considered in the presented results.

For post-analysis, the movements of the body segments were recorded with the OptiTrack system: rigid bodies of markers were placed on the end-effector, forearm, elbow, upper-arm, right and left acromions and trunk. The movements of the hip and shoulder joints were also recorded with Inertial Measurement Units (IMU), one on the trunk and one on the arm.

\section{B. Results and analysis}

To compare CCC with the two other modes of control (natural $\mathrm{N}$ and locked $\mathrm{L}$ ), we studied their performance through two criteria: task performance and joint motions, detailed in several metrics. Task performance was assessed through the end-effector trajectory error (defined as the mean distance between the WAM ${ }^{\mathrm{R}}$ and the subject's end-effectors during the task). The duration of the task is not interesting here since it was imposed by the $\mathrm{WAM}^{\mathrm{R}}$ Arm, and set constant between repetitions and between subjects.

Joint motions were assessed through the shoulder and elbow angles and the displacement of the acromion from its initial (reference) position. The shoulder angle is the humerus aperture, angle between the hip-acromion vector and the upper-arm vector; the elbow angle is the joint flexion (see Figure 6(a)).

The four corners of the rectangular trajectory are at different distances and heights from the subject; joint motion strategies may thus vary with movement phases. Hence, joint metrics are given for each of these four phases. Since modes $\mathrm{N}$ and $\mathrm{L}$ are proposed in this experiment to represent a natural reference and a fullly compensated scenario, joint metrics are also defined to allow a straightforward comparison of CCC with these two cases. For shoulder and elbow, the ratio between the joint angles obtained with modes L or CCC and the natural ones, at the end of each phase, is computed. Mode $\mathrm{N}$ thus represents $100 \%$ (perfect motion). The acromion metric is defined, for each phase, as the ratio $\left(x-\max _{N}\right) /\left(\max _{L}-\max _{N}\right)$, with $x=\left\{\max _{N} ; \max _{L} ; \max _{C C C}\right\}$ and $\max _{i}$ the maximum acromion displacement with mode $\mathrm{i}(\mathrm{i}=\mathrm{N}, \mathrm{L}, \mathrm{CCC})$ during the phase. This thus gives $0 \%$ for mode $\mathrm{N}$ and $100 \%$ for mode $\mathrm{L}$.

Results are shown Figure 6(b) (the values used to draw the box plots are the average over trials for each subject). It can be observed that there is no difference between the end-effector trajectory error of the control modes. The three outliers (one per mode) are from the same participant, whose trajectory error varies by less than $0.5 \mathrm{~cm}$ between the modes. Whatever the control mode, the task is correctly realized. The prosthesis user always finds a way to achieve the goal; what can differ is the corresponding joint motions (human or prosthetic) and the exhibited compensations.

When analyzing shoulder ratio, we can see that the shoulder angle follows the natural motion for the two first phases, be it with modes CCC or L. At the end of the third phase, 


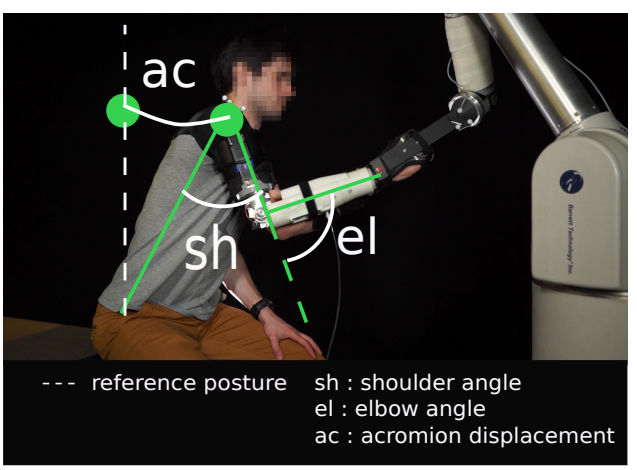

(a) Definition of joint metrics
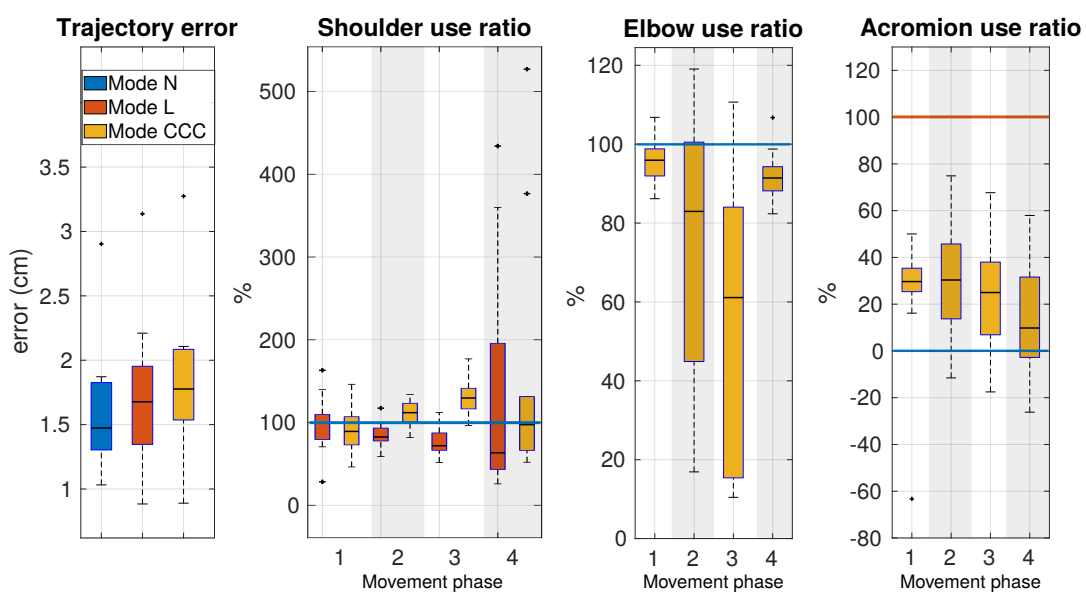

(b) Performance metrics

Fig. 6: Path tracking task performance. (a) Definition of joint measures. (b) Performance metrics of the three modes of control, averaged over trials for each subject. From left to right: trajectory error, shoulder use ratio, elbow use ratio, acromion use ratio.

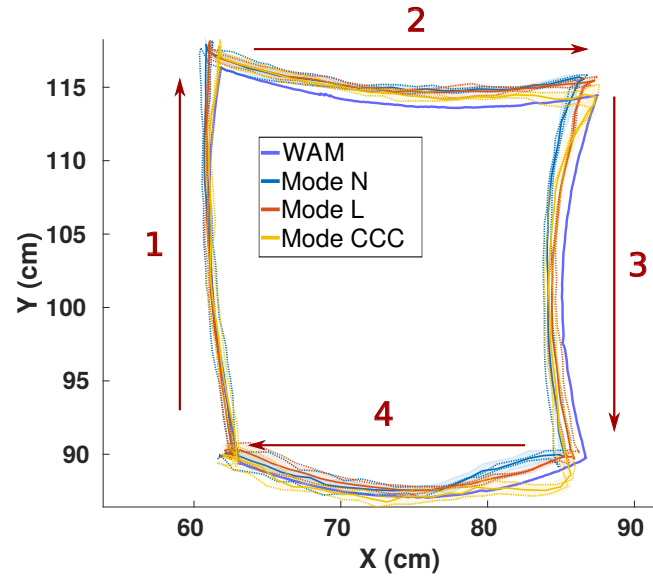

(a) End-effector trajectories

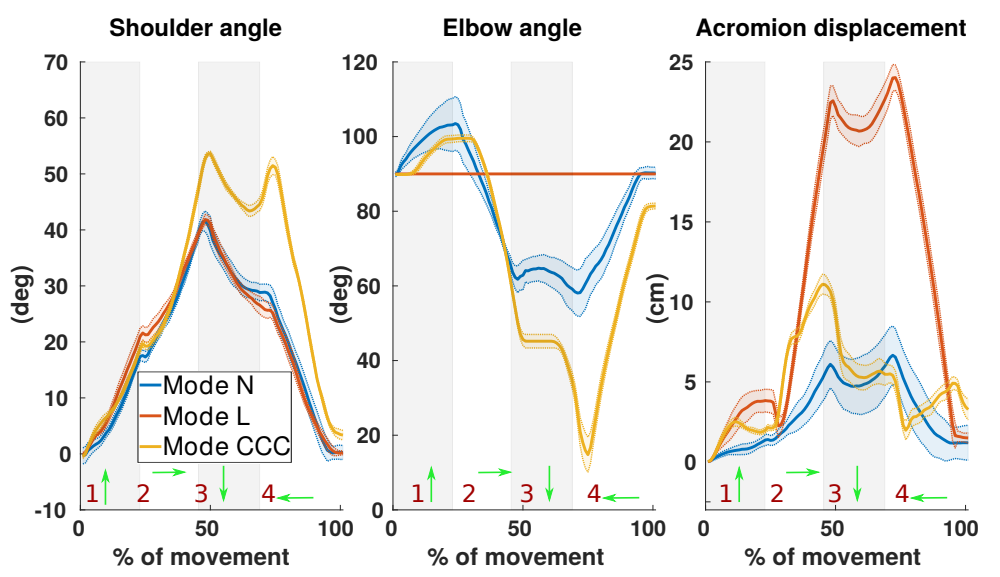

(b) Mean over trials and confidence interval of shoulder and elbow angles and acromion displacement.

Fig. 7: End-effector and joints trajectories from one subject, for the three control modes. The numbers and arrows indicate movement phases. (a) Comparison of user's end-effector and WAM's end-effector trajectories. For the user's, full line is the mean trajectory while dashed line is the standard deviation across the 5 trials. (b) Shoulder, elbow and acromion trajectories.

CCC induces a larger shoulder angle while mode $\mathrm{L}$ induces a smaller one. It comes back close to natural at the end of the fourth phase. The variation is high at this final step, with outlier data up to $500 \%$, due to very small values of shoulder angles for natural motions (close to zero); CCC and L final shoulder angles yet do not exceed $5 \mathrm{deg}$.

As for the elbow ratio, there is no value for mode $\mathrm{L}$ since there is no elbow motion. With CCC, the ratio is between 80 and $100 \%$ for the first and fourth phases; for the second and third ones, an over-extension and a high variability between subjects can be noticed. Elbow motions with CCC are thus similar to natural ones in close surroundings of the subject. For phases 2 and 3 (further zones to reach), we noticed that subjects tended to lean towards prosthetic side between the end of phase 2 and the beginning of phase 4 , in order to have a better vision of tracking error; this acromion displacement led to important elbow extension. The high variability is mainly due to the degree of leaning that differs between subjects which leads to more or less elbow extension. This points out the fact that trunk motions are not always compensatory but can also be functional (e.g. to position the head). Our approach does not yet separate these two situations. Larger shoulder angle at the end of phase 3 with CCC may be linked to this elbow over-extension, while the less large angle induced by mode L may be linked to the elbow still flexed at 90 deg whereas the natural one is more extended. Indeed, shoulder and elbow motions are connected to achieve the task; an over-extension of the elbow may thus be corrected by a higher shoulder angle to place the end-effector in a desired position. Once our approach will distinguish functional and compensatory roles of trunk and scapula, shoulder and elbow motions with CCC shall be close 
to natural ones during the entire task. This was already the case for participants who did not lean (or leant less) towards the prosthetic side in the third movement phase. The apparent inter-subject differences for the elbow and acromion metrics might also point out the need for a finer tuning of the control law parameters, although the small variability observed for phases 1 and 4 rather tends to discriminate this option.

The acromion ratio first shows a great reduction of compensatory motions with CCC compared to mode L (more than $60 \%$ on average). Then, we observe that body compensations are exhibited at the beginning of the task and decrease progressively until joining natural values.

Figure 7, showing end-effector, joints and acromion trajectories (mean +/- standard deviation) of one subject, confirms all these results. On Figure 7(a), we see that the task is correctly performed with the three control modes; on Figure 7(b), we see that shoulder and elbow angles with CCC follow natural values, except during phase 3 where there is an elbow over-extension which may induced the shoulder higher aperture. The over-extension is not as high for every subject. The acromion displacement with CCC is well reduced compared to mode $\mathrm{L}$ and very close to the natural one. Figure 7 also illustrates the small variability between trials, be it for end-effector or joint trajectories, whatever the control mode. After ten training trials only, human and prosthesis motions with $\mathrm{CCC}$ are as repeatable as human motions.

This experiment confirms that naive subjects can achieve a slow positioning task with $\mathrm{CCC}$ while intuitively mobilizing the device (i.e. without any instructions on how to move, nor any explanations on the prosthesis controller behavior). Exhibited trunk and scapula motions are small enough not to be considered as troublesome but large enough to move the prosthesis. It is also noteworthy that the same controller parameters, $\lambda$ and $q_{0}$, were used for all subjects. Even if a finer tuning around the values found in Section IV could be performed, there is no critical need for algorithm training or complex personalization.

\section{Preliminary test With an Amputee}

CCC response has been studied in details on ten ablebodied subjects to verify its implementation feasibility. We completed this study by performing the same experiment with a congenital amputee.

\section{A. Protocol}

The participant performed a path tracking task similar as in section $\mathrm{V}$. The dimensions of the rectangular trajectory were smaller than for able-bodied subjects, to adapt it to the reduced accessible space of the amputee, due to the socket (see Figure 8). The elbow joint of the prosthesis was successively controlled by two modes:

1) myoelectric control (MYO): a conventionnal myoelectric control with two electrodes (one on the biceps and the other on the triceps) was used to control the elbow flexion/extension. MYO controls the angular velocity with a trapezoidal profile. The participant was not used

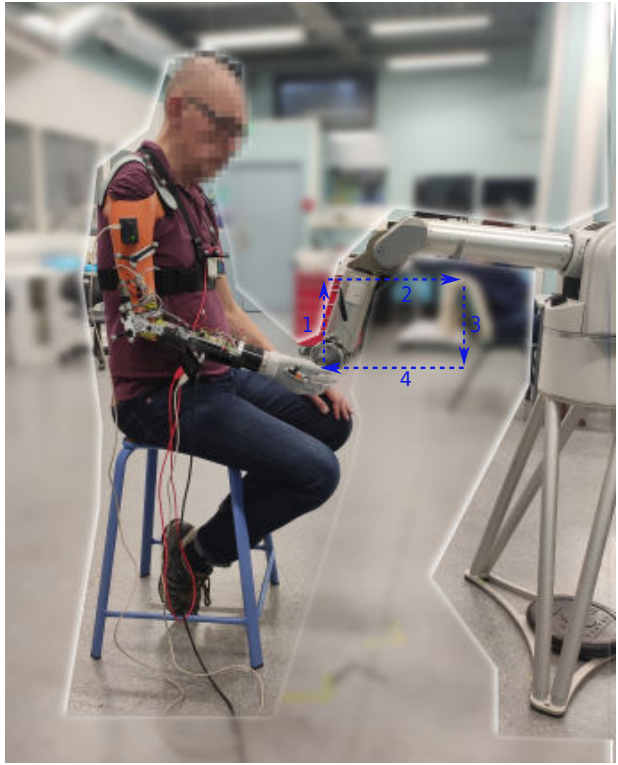

Fig. 8: Path tracking task for the amputated participant.

to myoelectric control because he began to wear a prosthesis shortly before the experiment. He had tested this mode only twice, in a different context, few days before the experiment;

2) Compensations Cancellation Control (CCC): $q_{0}$ was set to $5 \mathrm{deg}$ and $\lambda$ to $2 \mathrm{~s}^{-1}$.

The wrist and hand were locked. The task was repeated five times with each control mode and there was no specific training with $\mathrm{CCC}$ (the participant discovered this control mode during the first trial).

\section{B. Results and analysis}

Figure 9 shows the end-effector, shoulder, elbow and acromion trajectories, averaged over the five trials for the path tracking task performed by the amputee participant. It is completed by Figure 10 which shows performance metrics. As there is no natural reference here, shoulder, elbow and acromion metrics are not a comparison ratio but the final angles at the end of each movement phase and the maximum acromion displacement during each phase.

First, we can note that the trajectory error is similar between the two control modes (Figure 10(a)), which confirms what was observed with able-bodied participants: the prosthesis user adapts and manages to perform the task whatever the control mode, but the comfort and easiness of the motion can change. It could seem on Figure 9(a) that the end-effector trajectory with MYO is closer to the WAM trajectory than CCC, but this Figure only gives the spatial error and does not illustrate the possible time delay between the prosthesis' and the WAM's end-effector, which is taken into account in the trajectory error metric. Although similar between the two control modes, the error is higher than with able-bodied subjects, possibly because this participant did not have any familiarization neither with MYO nor with CCC.

Concerning shoulder joint, Figure 9(b) shows that the amplitude is smaller than the one of the representative able-bodied 


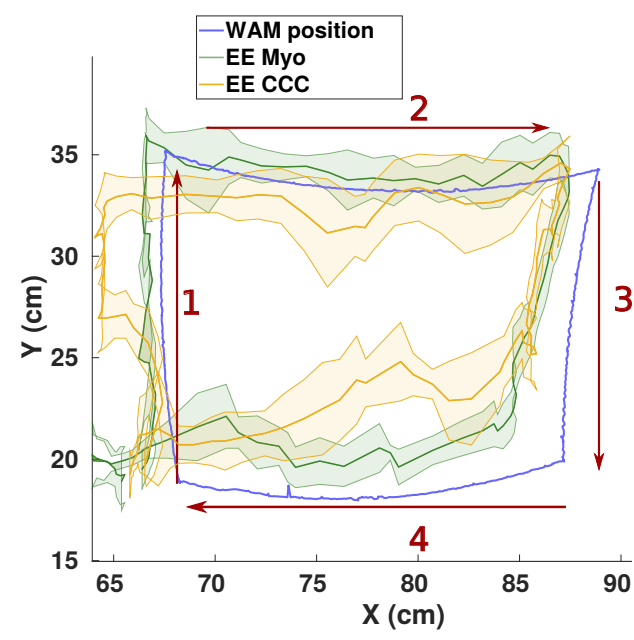

(a) End-effector trajectories
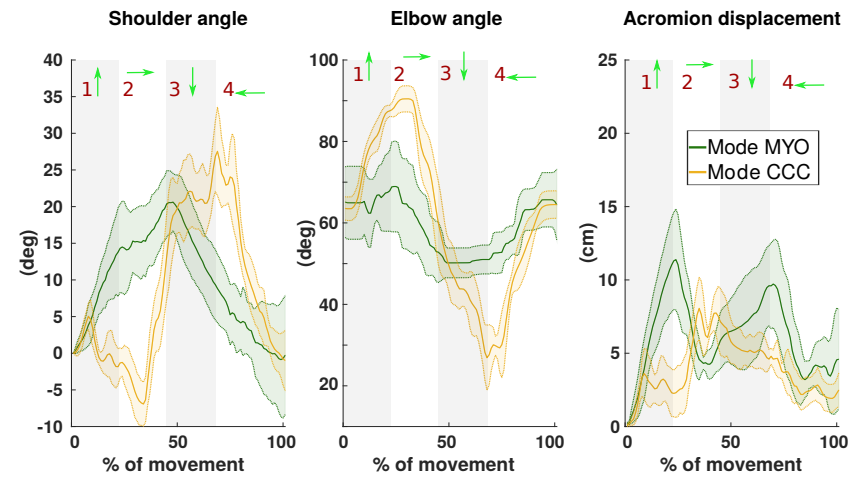

(b) Joints and acromion trajectories.

Fig. 9: End-effector and joint trajectories (mean $+/$ - standard deviation) with the two control modes for the amputated participant. The numbers and arrows indicate movement phases. (a) Comparison of user's and WAM's end-effector trajectories. (b) Shoulder, elbow and acromion trajectories.

subject, for the two modes. The temporal evolution is also a bit different; it has indeed been demonstrated that amputees' and able-bodied subjects' joints motion strategies can differ [35]. Figure 10(c) indicates that shoulder is not solicited at the same movement phases with MYO and CCC; this solicitation may depend on the elbow motion.

On Figure 9(b) and Figure 10(d), we can observe that the prosthetic elbow is much more mobilized with CCC than with $\mathrm{MYO}$, and that the angular trajectory with $\mathrm{CCC}$ is similar to the one of the representative able-bodied subject. As for the acromion, it is clear that its displacement is enhanced with MYO, in all movement phases (Figure 10(e)), meaning that the participant compensates more when using MYO than when using CCC. With the first one, the subject thus underuses the prosthetic elbow and prefers to move his hand with body compensations. This confirms that, when not familiar with MYO, a prosthesis user does not intuitively mobilize the device, but rather uses body motions, to realize a given task. Comparatively, $\mathrm{CCC}$ allows to mobilize the prosthetic joint (here the elbow), with minimized compensatory motions. This mobilization of the device is intuitive in the sense that no specific knowledge nor learning on the prosthetic control is required.

Figure 10(b) adds a new metric, to evaluate another benefit of the continuous human-prosthesis coupling: motion smoothness. Indeed, Auxiliary Signal Control, like myoelectric, requires the user to constantly adjust the prosthesis position by sending a new signal each time (here a new muscle contraction), which can induce more jerky movements than the closed loop control of CCC. Motion smoothness is evaluate here with the spectral arc length of the elbow angular velocity [36], computed for each movement phase and totaled over the four phases (see Figure 10(b); the more negative, the less smooth). We can see that prosthetic motions with CCC are smoother than the ones with MYO. From a more subjective point of view, the participant also reported that prosthetic motions with CCC seems more natural to him and induced less muscular fatigue.

A last thing to notice is that the controller parameters, $\lambda$ and $q_{0}$, were the same for able-bodied and amputated participants. CCC tuning is thus robust over subjects - a finer tuning could yet be suggested to find the most adequate $\left(\lambda, q_{0}\right)$ couple allowing the smoothest joint trajectory for each subject-.

\section{STUDY LIMITATIONS AND FUTURE WORKS}

These first results on $\mathrm{CCC}$ are promising for prostheses control but they are only preliminary and appeal for more exploration of the concept.

First, CCC was tested on only one DOF. A next step is to add more robotic DOF - apart from the hand whose grasping function must be covered distincly. The control principles should not be significantly modified and we expect to find here a simple and practical solution to the problem of coordinated control of a manipulator's joints (problem described in [37]). Some possible drawbacks arise from the fact that, in order to first develop the concept of $\mathrm{CCC}$, we focus mainly on issues related to control and coupling. We did not focus neither on the extraction of body compensations nor on the reference posture definition. The feasibility to measure and extract compensations in everyday life environment is a first important potential obstacle. Concerning the measure, we used a motion capture system, which can hardly be transferred out of a lab environment. For future developments, we will employ IMU: the positions of the end-effector and the acromion, required to compute the control input, can be reconstructed with a simple set of two IMU. With one IMU attached to the trunk and one on the upper arm, we could extract the trunk orientation as well as the relative humerus/trunk angle. Along with the angle of the prosthetic joints obtained through the encoder and some anatomical data of the user, the current positions of the end-effector and the acromion in the users's trunk frame can be derived [38], [39].

Concerning the identification of compensatory body positions, a major limitation was that the reference posture was defined as the initial position of the subjects and was fixed during the whole experiment. This solution does not allow identifying whether joints motions are compensatory or functional, since 


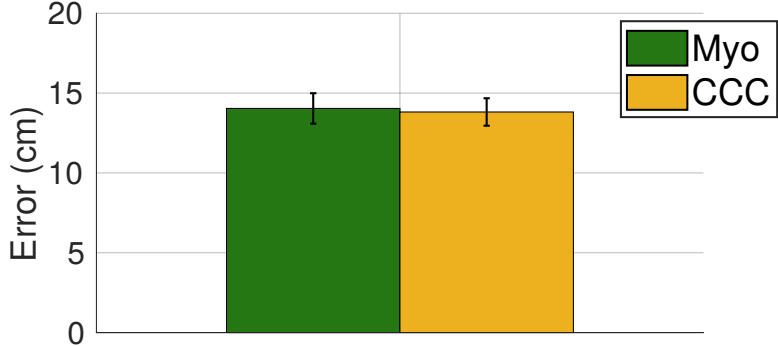

(a) Trajectory error

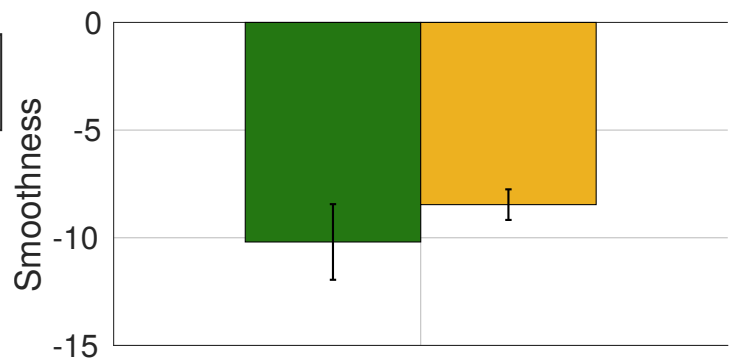

(b) Smoothness

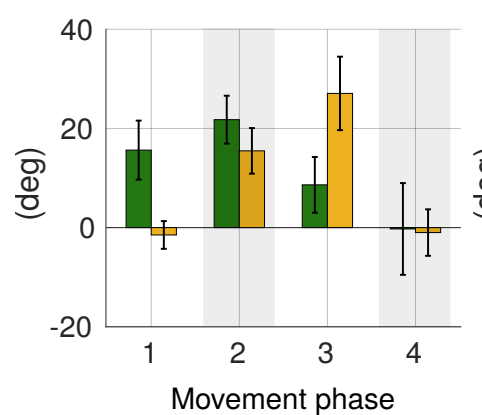

(c) Shoulder final angle at the end of each phase

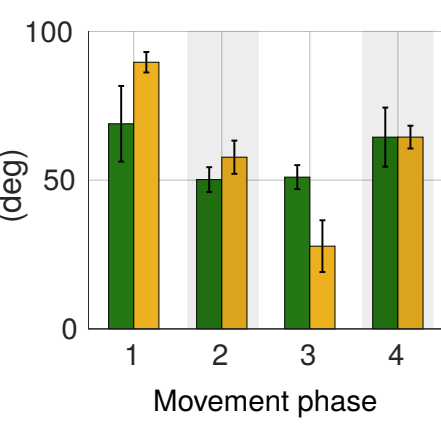

(d) Elbow final angle at the end of each phase

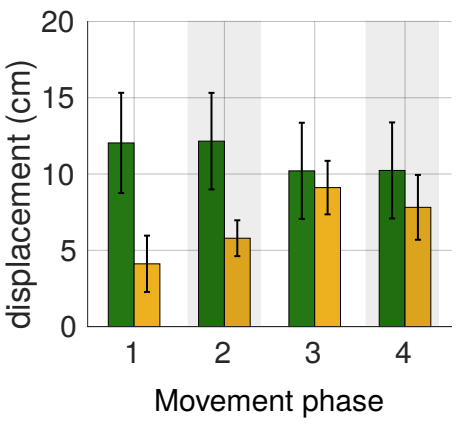

(e) Maximum acromion displacement for each phase

Fig. 10: Results of the path tracking task performed by the amputee participant. Metrics are averaged over the five trials for each mode.

every motion of the acromion is considered as a compensation, while it is not always the case. The reference posture can indeed vary with the context; it does not depend on the task itself but on the natural motions required to perform it. To tackle this issue, we are considering to develop a human model from which it could be recognized, in real time, whether the joint is compensatory or functional at the moment, and consequently trigger CCC.

In the presented work, CCC was compared to myoelectric control, with only one amputated subject. While the fact that the subject was naive to MYO was a good thing to assess the ease of discovering prosthetic control modes, this was also a limitation: a comparison of the two control modes with experienced subjects would be necessary to highlight the steady-state performance and thus the real ease of use of CCC in comparison to other control modes. This will be a point to assess in a future study. CCC could have also been compared to Motion Completion Control but the latter does not allow to perform a slow task like path tracking (it has only been developed for fast reaching motions). A new experiment can thus be set-up to both compare CCC to Motion Completion Control and study the feasibility to use $\mathrm{CCC}$ on tasks with different dynamics. Another supplementary work would be to analyze the cognitive load required by each control approach. It can be assumed that the generation of the auxiliary signal as well as the correction by the user only increase the mental charge; Auxiliary Signal Control as well as Motion Completion Control would thus be more demanding than Compensations Cancellation Control, but this is to be verified.

Finally, in Section IV, the human-prosthesis coupling, created by the device servoing on body compensations, was only illustrated with the controller tuning. It could be of interest to conduct a thorough stability analysis of the coupled system.

\section{CONCLUSION}

While the issues of posture-tracking and definition of reference body position in everyday life environment have been dismissed for the present study, the control paradigm based on posture correction presented here is validated. It allows a natural and simple command of an elbow prosthesis. After a few trials and without any explanation on the device's control, subjects manage to implicitly control the prosthesis to perform the tasks, while staying in a non-compensatory posture. Another important feature is that the controller has only two scalar parameters: an integral gain and a threshold (deadzone). After a tuning on one able-bodied subject, they were kept constant for all the participants, able-bodied or amputated, emphasizing the simplicity and robustness of the concept. It could yet be worthwhile to study whether a finer tuning for each user would allow for enhanced performance. Since the error defined to close the control loop is userbased, we believe that it will be possible to perform multiple tasks with the same control law, as long as they use similar compensatory motions.

Moreover, while tested on a prosthesis, the use of CCC is not 
limited to one kind of assistive device only. Many possible applications exist, be it for people living with disability or even for operators in professional context: upper limb exoskeletons, supernumerary arm, etc. To the authors' knowledge, there is no other existing control scheme apart CCC that, without any information on the task for the robot, continuously controls in real-time both the distal robotic joints of the assistive device and its user's posture. In that sense, the use of posture, combined with a feedback in the prosthesis control loop, opens new perspectives for prosthetic control.

\section{ACKNOWLEDGMENTS}

We would like to thank Dr. Jozina de Graaf, from Institut des Sciences du Mouvement at Aix-Marseille University, who helped us on the statistical analysis.

This work was supported by the ANR-BYCEPS, ANR-18CE19-0004.

\section{REFERENCES}

[1] S. Jönsson, K. Caine-Winterberger, and R. Brånemark, "Osseointegration amputation prostheses on the upper limbs: methods, prosthetics and rehabilitation," Prosthet. Orthot. Int., vol. 35, no. 2, pp. 190-200, Jun. 2011.

[2] C. Piazza, M. Catalano, S. Godfrey, M. Rossi, G. Grioli, M. Bianchi, K. Zhao, and A. Bicchi, "The SoftHand Pro-H: A hybrid bodycontrolled, electrically powered hand prosthesis for daily living and working," IEEE Robot. Autom. Mag., vol. 24, pp. 87-101, 2017.

[3] L. Resnik, S. L. Klinger, and K. Etter, "The deka arm: Its features, functionality, and evolution during the veterans affairs study to optimize the DEKA arm," Prosthet.Orthot. Int., vol. 38, pp. 492-504, 2014.

[4] F. Cordella, A. L. Ciancio, R. Sacchetti, A. Davalli, A. G. Cutti, E. Guglielmelli, and L. Zollo, "Literature review on needs of upper limb prosthesis users," Front. Neurosci., vol. 10, 2016.

[5] C. Castellini, P. Artemiadis, M. Wininger, A. Ajoudani, M. Alimusaj, A. Bicchi, B. Caputo, W. Craelius, S. Dosen, K. Englehart, D. Farina, A. Gijsberts, S. B. Godfrey, L. Hargrove, M. Ison, T. Kuiken, M. Marković, P. M. Pilarski, R. Rupp, and E. Scheme, "Proceedings of the first workshop on peripheral machine interfaces: Going beyond traditional surface electromyography," Front. Neurorobot., vol. 8, pp. $1-17,2014$.

[6] D. Farina, N. Jiang, H. Rehbaum, A. Holobar, B. Graimann, H. Dietl, and O. Aszmann, "The extraction of neural information from the surface emg for the control of upper-limb prostheses: Emerging avenues and challenges," IEEE Trans. Neural Syst. Rehabilitation Eng., vol. 22, no. 4, pp. 797-809, 2014.

[7] P. Beckerle, G. Salvietti, R. Unal, D. Prattichizzo, S. Rossi, C. Castellini, S. Hirche, S. Endo, H. Amor, M. Ciocarlie, F. Mastrogiovanni, B. Argall, and M. Bianchi, "A human-robot interaction perspective on assistive and rehabilitation robotics," Front. Neurorobot., vol. 11, 2017.

[8] A. Tabor, S. Bateman, and E. Scheme, "Evaluation of myoelectric control learning using multi-session game-based training," IEEE Trans. Neural Syst. Rehabil. Eng., vol. 26, pp. 1680-1689, 2018.

[9] I. Vujaklija, D. Farina, and O. Aszmann, "New developments in prosthetic arm systems," Orthop. Res. Rev., vol. 8, pp. 31-39, 2016.

[10] M. Velliste, S. Perel, M. C. Spalding, A. S. Whitford, and A. B. Schwartz, "Cortical control of a prosthetic arm for self-feeding," Nature, vol. 453, pp. 1098-1101, 2008.

[11] L. R. Hochberg, D. Bacher, B. Jarosiewicz, N. Y. Masse, J. D. Simeral, J. Vogel, S. Haddadin, and J. Liu, "Reach and grasp by people with tetraplegia using a neurally controlled robotic arm," Nature, vol. 485, pp. 372-377, 2012.

[12] L. Resnik, S. L. Klinger, K. Etter, and C. Fantini, "Controlling a multidegree of freedom upper limb prosthesis using foot controls: User experience," Disabil. Rehabil. Assist. Technol., vol. 9, pp. 318-329, 2014.

[13] A. Jackowski, M. Gebhard, and R. Thietje, "Head motion and head gesture based robot control: A usability study," IEEE Trans. Neural Syst. Rehabil. Eng., vol. 26, pp. 161-170, 2017.

[14] D. Graupe, "Control of an artificial limb in three degrees of freedom," Bull. Prosthet. Res., vol. 18, pp. 25-39, 1972.
[15] E. Abdi, E. Burdet, M. Bouri, and H. Bleuler, "Control of a supernumerary robotic hand by foot: An experimental study in virtual reality," PLoS One, vol. 10, 2015.

[16] M. Atzori and H. Müller, "Control capabilities of myoelectric robotic prostheses by hand amputees: A scientific research and market overview," Front. Syst. Neurosci., vol. 9, 2015.

[17] G. Li, A. Schultz, and T. Kuiken, "Quantifying pattern recognitionbased myoelectric control of multifunctional transradial prostheses," IEEE Trans. Neural Syst. Rehabil. Eng., vol. 18, pp. 185-192, 2010.

[18] S. Iftime, L. Egsgaard, and M. Popovic, "Automatic determination of synergies by radial basis function artificial neural networks for the control of a neural prosthesis," IEEE Trans. Neural Syst. Rehabil. Eng., vol. 13, pp. 482-489, 2005.

[19] M. Merad, E. de Montalivet, M. Legrand, E. Mastinu, M. Ortiz-Catalan, A. Touillet, N. Martinet, J. Paysant, A. Roby-Brami, and N. Jarrassé, "Assessment of an automatic prosthetic elbow control strategy using residual limb motion for transhumeral amputated individuals with socket or osseointegrated prostheses," IEEE Trans. Med. Robot. Bionics, 2020.

[20] M. L. Latash, "Motor synergies and the equilibrium-point hypothesis," Motor Control, vol. 14, no. 3, pp. 294-322, 2010.

[21] T. Bockemühl, N. F. Troje, and V. Dürr, "Inter-joint coupling and joint angle synergies of human catching movements," Hum. Mov. Sci., vol. 29, no. 1, pp. 73-93, 2010.

[22] C. Gambrell, "Overuse syndrome and the unilateral upper limb amputeeconsequences and prevention," J. Prosthet. Orthot., vol. 20, pp. 126-132, 2008.

[23] K. Østlie, R. J. Franklin, O. H. Skjeldal, A. Skrondal, and P. Magnus, "Musculoskeletal pain and overuse syndromes in adult acquired major upper-limb amputees," Arch. Phys. Med. Rehabil., vol. 92, pp. 19671973, 2011.

[24] S. L. Carey, M. Jason Highsmith, M. E. Maitland, and R. V. Dubey, "Compensatory movements of transradial prosthesis users during common tasks," Clin. Biomech., vol. 23, pp. 1128-1135, 2008.

[25] A. J. Metzger, A. W. Dromerick, R. J. Holley, and P. S. Lum, "Characterization of compensatory trunk movements during prosthetic upper limb reaching tasks," Arch. Phys. Med. Rehabil., vol. 93, pp. 2029-2034, 2012.

[26] A. Hussaini, A. Zinck, and P. Kyberd, "Categorization of compensatory motions in transradial myoelectric prosthesis users," Prosthet. Orthot. Int., vol. 41, pp. 286-293, 2016.

[27] A. Shafti, A. Ataka, B. Urbistondo Lazpita, A. Shiva, H. Wurdemann, and K. Althoefer, "Realtime robot assisted ergonomics," in Proc. IEEE Int. Conf. Robot. Autom., 2019, pp. 1975-1981.

[28] L. Peternel, W. Kim, J. Babi, and A. Ajoudani, "Towards ergonomic control of human-robot co-manipulation and handover," in Proc. IEEE RAS Int. Conf. Humanoid Robots, 2017, pp. 55-60.

[29] A. M. Zanchettin, E. Lotano, and P. Rocco, "Collaborative robot assistant for the ergonomic manipulation of cumbersome objects," in 2019 IEEE/RSJ Int. Conf. on Intelligent Robots and Systems (IROS), 2019.

[30] M. Legrand, E. de Montalivet, F. Richer, N. Jarrasse, and G. Morel, "Reciprocal Kinematic Control: using human-robot dual adaptation to control upper limb assistive devices," in Proc. Hamlyn Symposium on Medical Robotics, 2019, pp. 87-88.

[31] M. Cirstea and M. Levin, "Compensatory strategies for reaching in stroke," Brain, vol. 123, pp. 940-953, 2000.

[32] J. Perry, J. Rosen, and S. Burns, "Upper-limb powered exoskeleton design," IEEE/ASME Trans. Mechatronics, vol. 2, pp. 408-417, 2007.

[33] S. Hignett and L. McAtamney, "Rapid entire body assessment (REBA)," Appl. Ergon., vol. 31, no. 2, pp. 201-205, 2000.

[34] D. Kee and W. Karwowski, "LUBA: An assessment technique for postural loading on the upper body based on joint motion discomfort and maximum holding time," Appl. Ergon., vol. 32, pp. 357-366, 2001.

[35] M. Merad, E. de Montalivet, A. Touillet, N. Martinet, A. Roby-Brami, and N. Jarrassé, "Can we achieve intuitive prosthetic elbow control based on healthy upper limb motor strategies?" Front. Neurorobot., vol. 12, 2018.

[36] S. Balasubramanian, A. Melendez-Calderon, and E. Burdet, "A robust and sensitive metric for quantifying movement smoothness," IEEE Trans. Biomed. Eng., vol. 59, pp. 2126-2136, 2012.

[37] D. Whitney, "Resolved motion rate control of manipulators and human prostheses," IEEE Trans. Man-Mach. Syst., vol. 10, pp. 47-53, 1969.

[38] H. Zhou, H. Hu, and Y. Tao, "Inertial measurements of upper limb motion," Med. Biol. Eng. Comput., pp. 479-487, 2006.

[39] M. El-Gohary and J. McNames, "Shoulder and elbow joint angle tracking with inertial sensors," IEEE Trans. Biomed. Eng., vol. 59, pp. 2635-2641, 2012. 
Mathilde Legrand photograph and biography not available at the time of publication.

Nathanaël Jarrassé photograph and biography not available at the time of publication.

Etienne de Montalivet photograph and biography not available at the time of publication.

Florian Richer photograph and biography not available at the time of publication.

Guillaume Morel photograph and biography not available at the time of publication. 